\title{
Indicadores: o que são, o que não devem ser e o que poderão ser
}

António Luz Pereira*

[A contratualização é um processo] essencial para o desenvolvimento e modernização do Serviço Nacional de Saúde.

Constantino Sakellarides

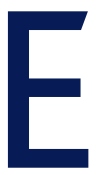
m 2005, concomitantemente com o lançamento da reforma dos cuidados de saúde primários (CSP) em Portugal, foi estabelecida como prioritária a introdução do processo de contratualização em todos os serviços do Serviço Nacional de Saúde. ${ }^{2}$

Podemos definir contratualização como um processo que visa a maximização dos ganhos em saúde, assim como a equidade e a sustentabilidade do sistema. Neste processo, um agente em representação da população identifica as suas necessidades em saúde, promove o planeamento e a operacionalização da prestação de serviços tendo em conta os recursos disponíveis e monitoriza o desempenho dos serviços quanto à qualidade dos cuidados e satisfação das necessidades em saúde. $^{3-4}$

Não se gere o que não se mede, não se mede o que não se define, não se define o que não se entende, não há sucesso no que não se gere.

W. Edwards Deming

Mas, muito antes do início da contratualização, o London Bills of Mortality 5 foi talvez o precursor dos processos de medição que a evolução do planeamento em saúde e de contratualização tornou essenciais. Com este percursor começou a dar-se importância aos resultados e desenvolveram-se instrumentos de medida

* Médico de família na USF Prelada, ACeS Porto Ocidental Departamento de Contratualização da ARS Norte

Doutorando em Investigação Clínica e Serviços de Saúde, Faculdade de Medicina da Universidade do Porto

Centro de Investigação em Tecnologias e Servicos de Saúde (CINTESIS), Faculdade de Medicina da Universidade do Porto

Editor da REVISTA PORTUGUESA DE MEDICINA GERAL E FAMILIAR para caracterizar o estado atual e objetivar as melhorias em saúde da população.

Esses instrumentos de medida foram sendo criados de forma a responder às necessidades dos diversos intervenientes, não só ao nível da atividade clínica, mas também para apoio à atividade de planeamento e gestão e facilitando ainda a prestação de contas com a população. Eles são usados para a medição dos recursos (inputs) e produção (outputs), mas também de resultados (outcomes) e impacto (impact). Enquanto os primeiros (inputs e outputs) são passíveis de ter a sua quantificação logo após a realização da atividade, nos de resultado e impacto, por vezes o efeito pode demorar a verificar-se.

Estes instrumentos de medida são aproximações matemáticas à realidade, não conseguindo transpor de forma objetiva toda a complexidade existente enxertada num conjunto de particularidades do mundo real. Esta dificuldade leva a que não possam ser consideradas metas definitivas, mas rumos de orientação, pelo que foi convencionado dar-lhes o nome de «indicadores».

A base numérica/matemática dos indicadores transmite alguma segurança numa ciência que Sir William Osler caracterizou como "a Medicina é uma ciência de incerteza e uma arte de probabilidade".

Mas, nos últimos anos, tem existido alguma «homofonia» entre prática clínica, indicador, desempenho e contratualização.

O contexto da saúde e as suas especificidades exigem uma leitura própria destes indicadores. Os cuidados de saúde são compostos por elementos mensuráveis, mas também por elementos cuja medição é impraticável. Apesar dos algoritmos simplificadores poderem ajudar na abordagem da doença, dificilmente poderão enquadrar a globalidade dos contextos e particularidades que caracterizam o doente no seu todo. ${ }^{6}$ Existe uma elevada incerteza nos processos em saúde e da sua conexão com os resultados obtidos ${ }^{7}$ e estima-se que cerca de $70 \%$ dos determinantes de saúde estejam fora do setor da prestação de cuidados em saúde. ${ }^{8-9}$ 
Num questionário realizado em 2014 nos EUA, ${ }^{10} \mathrm{em}$ que participaram cerca de 394 médicos, apenas $27 \%$ acreditam que os indicadores atuais representam moderadamente, ou de forma muito representativa, a qualidade dos cuidados que prestam.

Em abril de 2018, no New England Journal of MediCINE, MacLean e seus colaboradores ${ }^{11}$ referem que numa análise realizada a 86 indicadores, utilizando critérios da American College of Physicians, $37 \%$ foram considerados válidos e $35 \%$ não válidos e $28 \%$ com uma validade incerta, pelo que é necessário repensar toda a estrutura de indicadores e o modo como estes indicadores estão a ser integrados na atividade clínica.

A realidade nacional, expressa através do «Questionário Momento Atual», revela que uma grande percentagem dos coordenadores de Unidades de Saúde Familiar respondentes está em desacordo ou muito em desacordo relativamente à afirmação «Os indicadores têm fundamentação técnico-científica adequada» (64,8\% em $2013 / 14 ; 56,8 \%$ em $2014 / 15$ e $54,5 \%$ em $2016 / 17^{12}$ e $41,3 \%$ em $2017 / 18^{13}$ ). Aparentemente há menor crítica em relação à fundamentação técnica dos indicadores ao longo do tempo, podendo traduzir evolução nos indicadores ou uma habituação por parte dos coordenadores.

Não podemos achar que o indicador faz o nosso trabalho de negociar com o doente os seus alvos terapêuticos, nem por outro lado tentar atingir valores clinicamente pouco adequados para os nossos utentes para dar resposta ao indicador. Nesta, como noutras questões, é mandatório que a medicina seja centrada na pessoa e não em indicadores. Por outro lado, deveremos refletir o porquê dos valores atingidos, individualmente ou pela equipa, estarem afastados dos atingidos pelos nossos pares. A discussão e a partilha de experiência são essenciais, alicerçadas na governação clínica.

A utilização de um indicador deverá ter por base a convicção que este promoverá uma mudança na qualidade de processos dentro da organização, com melhores resultados em saúde e com o necessário suporte em evidência científica robusta.

É essencial que haja um compromisso entre a teoria e a prática e, para isso, é necessária uma ampla discussão para a melhoria e criação de indicadores entre os profissionais do terreno, gestores e investigadores.

Em Portugal já existe um caminho neste sentido, através da Portaria n. ${ }^{\circ}$ 212/2017, de 19 de julho, que cria a Comissão Técnica Nacional, tendo como missão "promover uma melhoria contínua e progressiva adaptação evolutiva e sustentada do modelo de contratualização e da sua operacionalização" e tem como competência validar os indicadores a serem utilizados na contratualização da matriz de indicadores dos CSP, definir os seus intervalos esperados e variação aceitável e elaborar propostas de melhoria da matriz de desempenho multidimensional.

Também a Coordenação Nacional para a Reforma dos CSP, no seu Plano Estratégico e Operacional, valoriza a contratualização e a matriz de indicadores. ${ }^{14}$

Existem atualmente algumas ferramentas que também nos possibilitam avaliar os indicadores. Um exemplo é o guia orientador INDICABEM, do Grupo Técnico Nacional da Governação Clínica e de Saúde nos Cuidados de Saúde Primários. ${ }^{15}$ Neste documento, de fácil leitura, é possível conhecer alguns critérios de apreciação de indicadores e desmistificar alguns conceitos. Também noutras indústrias e setores da economia é utilizado o conceito de Controlo Estatístico de Qualidade que, sendo uma forma de controlo de indicadores e processos, poderá ter replicação na área da saúde.

Existe ainda um longo caminho a percorrer no sentido de permitir um pensamento mais próximo da prática clínica real, em que os valores dos indicadores são o resultado desta prática e não o inverso.

Desta forma, a metodologia de contratualização iniciada em 2017, centrada nas atividades, veio dar mais um passo no sentido de encontrar indicadores que cada vez mais reflitam a qualidade da prática clínica e traduzam melhores ganhos em saúde.

No futuro será essencial trabalhar com indicadores que permitam ser instrumentos de decisão clínica, validados para a análise de resultados e com reconhecimento do seu interesse por parte dos profissionais. Para que isso aconteça, será indispensável incluir os profissionais no desenho da matriz de indicadores.

Para além da avaliação da satisfação dos utilizadores já observada ao nível dos CSP, é igualmente essencial dar uma voz cada vez mais forte aos cidadãos e incluir neste processo o seu próprio conceito de valor dos cuidados em saúde.

Só com participação ativa por parte de todos os intervenientes neste processo será possível encarar cada 
vez mais os indicadores não pela perspetiva do que eles nunca poderão ser, mas por todos os potenciais ganhos que deles poderão advir.

\section{REFERÊNCIAS BIBLIOGRÁFICAS}

1. Sakellarides C. Repensando a contratualização. Rev Port Saúde Pública. 2010;temático(9):5-6.

2. Monteiro BR, Pisco AM, Candoso F, Bastos S, Reis M. Cuidados primários em saúde em Portugal: 10 anos de contratualização com os serviços de saúde na Região de Lisboa [Primary healthcare in Portugal: 10 years of contractualization of health services in the region of Lisbon]. Cien Saude Coletiva. 2017;22(3):725-36. Portuguese, english

3. Gardner K, Davies GP, Edwards K, McDonald J, Findlay T, Kearns R, et al. $A$ rapid review of the impact of commissioning on service use, quality, outcomes and value for money: implications for Australian policy. Aust J Prim Health. 2016;22(1):40-9.

4. Robinson S, Dickinson H, Durrington L. Something old, something new, something borrowed, something blue? Reviewing the evidence on commissioning and health services. Aust J Prim Health. 2016;22(1):9-14.

5. Johansson SR. When numbers began to count for health policy: a review essay. Popul Dev Rev. 2003;29(4):715-43.

6. Starfield B, Shi L, Macinko J. Contribution of primary care to health systems and health. Milbank Q. 2005;83(3):457-502.

7. Patterson ME, Hernandez AF, Hammill BG, Fonarow GC, Peterson ED, Schulman KA, et al. Process of care performance measures and longterm outcomes in patients hospitalized with heart failure. Med Care. 2010;48(3):210-6.

8. McGinnis JM, Williams-Russo P, Knickman JR. The case for more active policy attention to health promotion. Health Aff (Millwood). 2002;21(2):78-93.
9. George F. Sobre determinantes da saude [Internet]. Lisboa: Direção-Geral da Saúde; 2011. Available from: https://www.dgs.pt/director-ge$\mathrm{ral} /$ francisco-george/publicacoes-de-francisco-george.aspx

10. Casalino LP, Gans D, Weber R, Cea M, Tuchovsky A, Bishop TF, et al. US physician practices spend more than $\$ 15.4$ billion annually to report quality measures. Health Aff (Millwood). 2016;35(3):401-6.

11. MacLean $\mathrm{CH}$, Kerr EA, Qaseem A. Time out: charting a path for improving performance measurement. N Engl J Med. 2018;378(19):1757-61.

12. Biscaia A, Fehn AC, Cardeira R, Pereira A. O momento actual da reforma dos cuidados de saúde primários em Portugal: as oito edições de 2009 a 2017. Coimbra: Mar da Palavra; 2017. ISBN 9789728910761

13. Biscaia A, Pereira A, Cardeira R, Fehn AC, Pinto TV. O momento atual da reforma dos cuidados de saúde primários em Portugal, 2017/2018: questionário aos coordenadores de USF. Lisboa: Associação Nacional de Unidades de Saúde Familiar; 2018.

14. Gabinete do Secretário de Estado Adjunto e da Saúde. Reforma do Serviço Nacional de Saúde - Coordenação nacional para os cuidados de saúde primários: plano estratégico e operacional. Lisboa: GSEAS; 2016. Available from: https://www.sns.gov.pt/wp-content/uploads/2016/02/ Plano_Estrategico_e_Operacional.pdf

15. Felício MM, Machado V. INDICABEM - Uma ferramenta para a apreciação crítica de indicadores em saúde: guia orientador (documento em progresso) [Internet]. Lisboa: Serviço Nacional de Saúde; 2017. Available from: https://bicsp.min-saude.pt/pt/biblioteca/Biblioteca/IndicaBem.pdf

\section{ENDEREÇO PARA CORRESPONDÊNCIA}

António Luz Pereira

E-mail: antoniolpereira@gmail.com

http://orcid.org/0000-0002-8290-5297 